\title{
El formalismo de contextos generalizados y la medición cuántica*
}

Formalism of Generalized Contexts and Quantum Measurement

Marcelo Losada ${ }^{\dagger}$

Roberto Laura

\begin{abstract}
Resumen
En este trabajo se describe el proceso de medición de un sistema cuántico como una interacción entre dos sistemas físicos: el sistema a medir y el aparato de medición. Ambos sistemas forman un sistema compuesto y su evolución temporal está determinada únicamente por la ecuación de Schrödinger, es decir, no hay postulado de colapso. La descripción del proceso de medición se realiza utilizando un formalismo de historias cuánticas denominado formalismo de Contextos Generalizados. Este formalismo permite expresar, a través de la probabilidad condicional, la correlación entre las propiedades correspondientes al observable medido, antes de la medición, y las propiedades correspondientes a la variable puntero del aparato, después de la medición. Utilizando este formalismo, se discuten dos problemas centrales de la medición: el problema de la lectura definida y el problema de la base privilegiada.
\end{abstract}

Palabras clave: fundamentos de cuántica - historias cuánticas - medición cuántica

\begin{abstract}
In this paper, the measurement process of a quantum system is described as an interaction of two physical systems: the system to be measured and the measuring instrument. Both systems form a composite system and its temporal evolution is determined solely by the Schrödinger equation, i.e., there is no collapse postulate. The description of the measurement process is performed using a formalism of quantum histories called formalism of Generalized Contexts. This formalism allows to express, using the conditional probability, the correlation between the properties corresponding to the measured observable, before the measurement, and the properties corresponding to the pointer variable of the measurement instrument, after the measurement. Using this formalism, two central problems of quantum measurement are discussed: the problem of definite outcome and the problem of preferred basis.
\end{abstract}

Keywords: quantum foundations - quantum histories - quantum measurement

\footnotetext{
* Recibido: 29 de mayo de 2018. Aceptado con revisiones: 25 de agosto de 2018.

† Universidad de Buenos Aires/CONICET, Argentina. Para contactar al autor, por favor, escribir a: marcelolosada@yahoo.com.

* Universidad Nacional de Rosario, Argentina. Para contactar al autor, por favor escribir a: rlaura@fceia.unr.edu.ar. Metatheoria 9(2)(2019): 83-94. ISSN 1853-2322. eISSN 1853-2330.

(c) Editorial de la Universidad Nacional de Tres de Febrero. Publicado en la República Argentina.
} 


\section{Introducción}

En la formulación ortodoxa de la mecánica cuántica los estados de un sistema físico tienen dos tipos de evoluciones temporales: la evolución de Schrödinger y la evolución por colapso. La primera se aplica a los procesos físicos ordinarios en los cuales no se realizan mediciones. La dinámica está gobernada por la ecuación de Schrödinger y la evolución temporal resultante es continua y determinista. La segunda se aplica a los procesos de medición. Cuando un sistema físico es sometido a una medición, el estado colapsa al autovector correspondiente al valor de la magnitud medida. A diferencia de la evolución de Schrödinger, la evolución por colapso es discontinua y no determinista.

Esta formulación tiene un notable éxito predictivo. Sin embargo, la distinción entre procesos físicos ordinarios y procesos de medición, cada uno gobernado por un tipo de evolución temporal diferente, no es satisfactoria desde un punto de vista teórico.

Por un lado, como únicamente se define la probabilidad de que una propiedad sea medida, no es aplicable a aquellos sistemas a los que no se les realizan mediciones. Por otro lado, como los aparatos de medición están compuestos en última instancia por las mismas partículas que el resto de los sistemas físicos, se espera que la medición no sea un proceso esencialmente diferente al resto de los procesos físicos. Por lo tanto, es deseable una teoría cuántica que describa a ambos tipos de procesos de la misma manera, utilizando únicamente la evolución de Schrödinger.

Estas dificultades teóricas motivaron a considerar a los procesos de medición como procesos ordinarios de interacción entre dos sistemas cuánticos: el sistema a medir y el aparato de medición. Esta manera de describir la medición es un paso necesario en la búsqueda de una interpretación realista de la mecánica cuántica que no describa únicamente los resultados de las mediciones (Laura \& Vanni 2008).

La descripción del proceso de medición como una interacción entre sistemas cuánticos involucra resolver la ecuación de Schrödinger del sistema físico compuesto por el sistema a medir y el aparato de medición. Para ello se debe tener en cuenta el hamiltoniano total del sistema compuesto, que incluye tres partes: el hamiltoniano del aparato, el hamiltoniano del sistema a medir y el hamiltoniano de interacción. Además, el estado inicial debe representar el hecho de que el aparato y el sistema a medir fueron preparados en forma independiente y que el aparato está listo para la medición.

Al resolver la ecuación de Schrödinger se obtiene como estado final una combinación lineal de autovectores de la variable indicadora. Sin embargo, experimentalmente se observa que al finalizar la medición la variable indicadora del aparato toma un valor definido. Por lo tanto, describir el proceso de medición sin hacer uso del postulado de colapso requiere poder dar cuenta de cómo se obtiene un valor definido de la variable puntero.

Otra cuestión relevante para describir la medición sin el postulado de colapso es la necesidad de relacionar el valor de la variable indicadora del aparato, un tiempo después de la medición, con la propiedad del sistema a medir, un tiempo antes de la medición (Ballentine 1998). Sin embargo, en la mecánica cuántica ortodoxa únicamente se pueden asignar probabilidades a propiedades a un mismo tiempo, no es posible asignar probabilidades a conjunciones o disyunciones entre propiedades a distintos tiempos.

Las teorías de historias cuánticas pretenden resolver las dificultades mencionadas previamente. En primer lugar, proveen una interpretación de la mecánica cuántica en la que los procesos de medición son tratados de la misma forma que el resto de los procesos físicos. La medición pierde su estatus privilegiado y pasa a ser descripta como una interacción física ordinaria entre un sistema a medir y un aparato de medición.

En segundo lugar, abandonan la idea de que los observables de un sistema físico tienen un valor definido únicamente si el vector de estado coincide con algún autovector del operador asociado a 
dicho observable. Según este enfoque, el vector de estado que surge de resolver la ecuación de Schrödinger no describe las propiedades actuales del sistema, sino que determina las probabilidades de que las propiedades posibles se actualicen.

En tercer lugar, extienden el formalismo estándar de la mecánica cuántica de modo tal de poder definir operaciones lógicas entre propiedades a distintos tiempos. Para ello introducen la noción de historia de un sistema físico, que generaliza a la noción de propiedad. Una historia se define como una secuencia de propiedades a distintos tiempos u operaciones lógicas entre ellas.

Si bien no existe una única teoría de historias cuánticas, sus fuertes similitudes justifican que se las englobe bajo el mismo nombre. Sus inicios se remontan a la década de 1980. Robert B. Griffiths (1984) presentó la primera versión de la teoría de Historias Consistentes. Tiempo después presentó algunas modificaciones de aquella versión (Griffiths 2002, 2013). Por otro lado, Roland Omnès (1988, 1994, 1999) publicó una serie de trabajos en los que contribuyó al desarrollo de esta teoría. Paralelamente, Murray Gell-Mann y James Hartle (1990) desarrollaron un formalismo de historias similar al de Historias Consistentes, denominado Historias Decoherentes. Años más tarde Roberto Laura, Leonardo Vanni y Marcelo Losada desarrollaron un formalismo alternativo de historias denominado formalismo de Contextos Generalizados, el cual se propone evitar algunas de las complicaciones de los enfoques anteriores (Laura \& Vanni 2008, 2009, Laura, Vanni \& Losada 2013).

En este trabajo se analizará el proceso de medición utilizando el formalismo de Contextos Generalizados. En la sección 2 se utiliza el formalismo de la mecánica cuántica ortodoxa para describir el proceso de medición como una interacción entre un sistema a medir y un aparato de medición. Luego, se presentan sus dos problemas centrales: el problema de lectura definida y el problema de la base privilegiada. En la sección 3 se resumen las ideas básicas del formalismo de Contextos Generalizados. En la sección 4 se aplica el formalismo para describir el proceso de medición y se muestra que su interpretación del vector de estado elimina el problema de la lectura definida. En la sección 5 se discute el problema de la base privilegiada y la posibilidad de que un aparato de medición mida más de un observable. Por último, en la sección 6 se presentan las conclusiones del trabajo.

\section{Descripción cuántica de la medición}

En esta sección se describe el proceso de medición como una interacción entre dos sistemas cuánticos: el sistema a medir y el aparato de medición. El sistema total está compuesto por ambos sistemas y su evolución temporal está determinada únicamente por la ecuación de Schrödinger, es decir, no hay postulado de colapso.

Sea un sistema $S$ a medir por un aparato de medición $A$. Al sistema total, compuesto por $S$ y por $A$, se lo denomina $S+A$. Los espacios de Hilbert del sistema $S$ y del aparato $A$ son $\mathcal{H}_{S}$ y $\mathcal{H}_{A}$, respectivamente. El espacio de Hilbert del sistema compuesto es el producto tensorial de los espacios de Hilbert del sistema $S$ y del aparato $A$, es decir, $\mathcal{H}_{S A}=\mathcal{H}_{S} \otimes \mathcal{H}_{A}$.

El proceso de medición del sistema $S$ por parte del aparato de medición $A$ se describe como una interacción entre ambos sistemas, cuya dinámica está dada por la ecuación de Schrödinger del sistema compuesto. El aparato $A$ mide a un observable $Q$ del sistema $S$ representado por el operador $\hat{Q}$, cuyos autovalores son los números $q_{i}$ y sus respectivos autovectores son $\left|q_{i}\right\rangle \in \mathcal{H}_{S}$ es decir, $\hat{Q}\left|q_{i}\right\rangle=q_{i}\left|q_{i}\right\rangle$. El conjunto de los autovectores $\left|q_{i}\right\rangle$ forma una base del espacio $\mathcal{H}_{S}$.

Si el aparato $A$ mide al observable $\hat{Q}$, entonces los autovectores $\left|q_{i}\right\rangle$ deben corresponderse, luego de la medición, con ciertos estados macroscópicos $\left|a_{i}\right\rangle \in \mathcal{H}_{A}$ del aparato $A$. Cada vector $\left|a_{i}\right\rangle$ representa a un estado del aparato accesible al observador, como por ejemplo el estado asociado a una posición de la aguja indicadora. Los vectores $\left|a_{i}\right\rangle$ son autovectores de un operador $\hat{A}$ definido sobre 
el espacio de Hilbert $\mathcal{H}_{A}$, es decir, $\hat{A}\left|a_{i}\right\rangle=a_{i}\left|a_{i}\right\rangle$. Al observable $\hat{A}$ se lo denomina variable puntero del aparato y los números $a_{i}$ son sus posibles valores.

Supondremos que el proceso de medición comienza en un tiempo $t_{1}$ y termina en otro tiempo $t_{2}>t_{1}$. Además, consideramos que el operador de evolución temporal $U\left(t_{2}, t_{1}\right)$ satisface

$$
\left|q_{i}\right\rangle\left|a_{0}\right\rangle \stackrel{U\left(t_{2}, t_{1}\right)}{\longrightarrow}\left|\phi_{i}\right\rangle\left|a_{i}\right\rangle
$$

donde:

i) $\left|a_{0}\right\rangle \in \mathcal{H}_{A}$ es el vector que representa al estado del aparato antes del proceso de medición (el cero de la variable indicadora),

ii) $\left|\phi_{i}\right\rangle \in \mathcal{H}_{S}$ es el estado del sistema $S$ en que se transforma el estado inicial $\left|q_{i}\right\rangle \in \mathcal{H}_{S}$, después del proceso de medición (si $\left|\phi_{i}\right\rangle=\left|q_{i}\right\rangle$, la medición se denomina ideal),

iii) $\left|a_{i}\right\rangle \in \mathcal{H}_{A}$ es un estado del aparato que es accesible al observador (por ejemplo, el estado asociado a una posición de la aguja indicadora).

La expresión (1) muestra que si el estado inicial del sistema $S$ es $\left|q_{i}\right\rangle$ y el del aparato es $\left|a_{0}\right\rangle$ (el cero de la variable indicadora), el proceso de interacción sistema-aparato pone en correspondencia al resultado de la medición $\left|a_{i}\right\rangle$ al tiempo $t_{2}$ con el estado $\left|q_{i}\right\rangle$ del sistema $S$ a un tiempo $t_{1}$ anterior a $t_{2}$.

Se debe notar que si antes de la medición el estado del sistema $S+A$ es de la forma $\left|\Psi_{t_{1}}\right\rangle=|\varphi\rangle\left|a_{0}\right\rangle$, donde $|\varphi\rangle=\sum_{i} c_{i}\left|q_{i}\right\rangle$, entonces, debido a la linealidad de la ecuación de Schrödinger, se obtiene que después de la medición el estado del sistema compuesto es $\left|\Psi_{t_{2}}\right\rangle=\sum_{i} c_{i}\left|q_{i}\right\rangle\left|a_{i}\right\rangle$. Es decir, en el caso general el estado final es una combinación lineal de estados macroscópicos del aparato de medición.

La interpretación ortodoxa considera que un observable tiene un valor definido únicamente si el estado del sistema coincide con un autovector del observable. En este caso, la variable puntero, representada por $\hat{A}$, tendría un valor definido $a_{i}$ solamente si $\hat{A}|\Psi\rangle=a|\Psi\rangle$. Por lo tanto, si el estado inicial del sistema $S+A$ es de la forma $\left|\Psi_{t_{1}}\right\rangle=|\varphi\rangle\left|a_{0}\right\rangle$, entonces, luego de la medición, no se obtendría un valor definido de la variable puntero. Esto contradice a lo que sucede en los laboratorios, donde cada vez que se realiza una medición se obtiene un resultado definido. A este problema se lo conoce como el problema de la lectura definida (problem of definite outcome).

Otro problema que surge al describir la medición como una interacción entre sistemas cuánticos es la ambigüedad en la elección de la base en la cual descomponer el estado final del sistema compuesto. En la formulación ortodoxa cada aparato de medición mide a un observable $Q$ del sistema. Luego de la medición el sistema colapsa al autovector correspondiente al autovalor medido de $Q$. En esta descripción de la medición no hay ambigüedad respecto a la base en la cual proyectar a los estados: cada aparato mide a un observable y la base que se debe usar es la base que diagonaliza a dicho observable. Cuando se abandona el postulado de colapso y se describe a la medición como una interacción entre sistemas cuánticos, surge la ambigüedad de la base en la cual descomponer el estado final del sistema compuesto. En general la descomposición del estado final no es única, sino que depende de la base elegida. Esta segunda dificultad se conoce como el problema de la base privilegiada (problem of preferred basis).

Una descripción coherente de la medición como proceso de interacción entre sistemas cuánticos debe dar cuenta de los problemas anteriores. En este trabajo se analizarán los dos problemas utilizando 
un formalismo de historias cuánticas denominado formalismo de Contextos Generalizados. En la siguiente sección se resumen las ideas principales de este formalismo. 


\section{Formalismo de Contextos Generalizados}

Las teorías de historias cuánticas surgieron como formulaciones alternativas de la mecánica cuántica ortodoxa, con la finalidad de resolver algunas de sus dificultades interpretativas. Por un lado, proveen una interpretación de la mecánica cuántica en la que los procesos de medición son tratados de la misma forma que el resto de los procesos físicos. La medición pierde su estatus privilegiado y pasa a ser descripta como una interacción física ordinaria entre un sistema a ser medido y un aparato de medición. Por otro lado, extienden el formalismo estándar de la mecánica cuántica de modo tal de poder definir operaciones lógicas entre propiedades a distintos tiempos.

Existen diferentes formalismos de historias cuánticas. Robert B. Griffiths (1984) presentó la teoría de Historias Consistentes. Tiempo más tarde, Roland Omnès publicó una serie de trabajos en los que contribuyó al desarrollo de esta teoría (Omnès 1988, 1994, 1999). Por otro lado, Murray Gell-Mann y James Hartle (1990) desarrollaron otro formalismo de historias llamado Historias Decoherentes. Años más tarde Roberto Laura, Leonardo Vanni y Marcelo Losada (2008, 2009, 2013) desarrollaron un formalismo alternativo de historias denominado formalismo de Contextos Generalizados. En este trabajo se utilizará el formalismo de Contextos Generalizados.

A continuación se resumen sus aspectos generales. En el apartado 3.1. se describen los contextos de propiedades ordinarios de la mecánica cuántica y en el apartado 3.2. se generalizan estas ideas y se presenta el formalismo de Contextos Generalizados.

\subsection{Contextos de propiedades}

En la mecánica cuántica cada sistema físico tiene asociado un espacio de Hilbert $\mathcal{H}$ y un operador Hamiltoniano $H$. Las propiedades de un sistema se presentan por proyectores ortogonales. Dada una propiedad $p$, se denomina $\Pi_{p}$ a su proyector correspondiente. Los estados del sistema se representan por operadores estadísticos y generalmente se denotan con la letra griega $\rho$.

En la representación de Schrödinger el operador de estado evoluciona en el tiempo según la ecuación de Liouville-von Neumann. La solución de esta ecuación se puede expresar a través del operador de evolución temporal dado por

$$
U\left(t^{\prime}, t\right)=e^{-\frac{i}{\hbar} H\left(t^{\prime}-t\right)}
$$

El operador $U\left(t^{\prime}, t\right)$ relaciona al operador de estado a un tiempo $t$ con el operador de estado a un tiempo $t^{\prime}$ de la siguiente manera:

$$
\rho_{t^{\prime}}=U\left(t^{\prime}, t\right) \rho_{t} U\left(t, t^{\prime}\right),
$$

donde $\rho_{t}$ y $\rho_{t}$, representan a los operadores de estado en los tiempos $t$ y $t^{\prime}$, respectivamente.

La mecánica cuántica asigna probabilidades a las propiedades de un sistema a partir de la regla de Born. Para cada tiempo $t_{i}$ la probabilidad está dada por

$$
\operatorname{Pr}_{t_{i}}(p)=\operatorname{Tr}\left(\rho_{t_{i}} \Pi_{p}\right)
$$

Sin embargo, no es posible asignar probabilidades a todas las propiedades simultáneamente. El conjunto de propiedades tiene estructura de reticulado ortocomplementado no distributivo. Para que la probabilidad esté bien definida hay que restringirla a un subreticulado distributivo de propiedades. Esto es equivalente a seleccionar conjuntos de propiedades representados por proyectores que conmutan entre sí. A los subreticulados distributivos de propiedades se los denomina contextos de propiedades (Mittelstaedt 1998, Cohen 1989).

Para obtener un contexto de propiedades $\mathcal{C}_{i}$ al tiempo $t_{i}$ primero se debe elegir un conjunto de propiedades atómicas $p_{i}^{k_{i}}\left(k_{i} \in \sigma_{i}\right.$ y $\sigma_{i}$ un conjunto de índices) que estén representadas por 
proyectores $\Pi_{i}^{k_{i}}$ que formen una descomposición proyectiva del espacio de Hilbert $\mathcal{H}$, es decir, que sean mutuamente ortogonales y sumen la identidad,

$$
\sum_{k_{i} \in \sigma_{i}} \Pi_{i}^{k_{i}}=I, \quad \Pi_{i}^{k_{i}} \Pi_{i}^{k_{i}^{\prime}}=\delta_{k_{i} k_{i}^{\prime}} \Pi_{i}^{k_{i}} .
$$

Luego, cualquier propiedad $p$ del contexto $\mathcal{C}_{i}$ se representa como una suma de proyectores de la descomposición proyectiva

$$
\Pi_{p}=\sum_{k_{i} \in \sigma_{p}} \Pi_{i}^{k_{i}}, \quad \text { con } \quad \sigma_{p} \subseteq \sigma_{i} .
$$

Por último, a partir de la regla de Born, se define la probabilidad sobre el contexto de propiedades $\mathcal{C}_{i}$.

En la representación de Schrödinger los estados evolucionan en el tiempo y las propiedades del sistema están fijas. Una representación alternativa es la de Heisenberg, en la cual sucede a la inversa: los estados están fijos y las propiedades evolucionan en el tiempo.

En esta última, se elige un tiempo de referencia $t_{0}$ para describir a los estados y la evolución temporal de las propiedades está dada por el operador de evolución temporal. Dada una propiedad $p$ al tiempo $t_{i}$, representada por el proyector $\Pi_{p, t_{i}}$, su evolución temporal está dada por

$$
\Pi_{p, t}=U\left(t, t_{i}\right) \Pi_{p, t_{i}} U\left(t_{i}, t\right) .
$$

La probabilidad de la propiedad $p$ al tiempo $t_{i}$ se escribe en términos del estado a un tiempo de referencia $t_{0}$ y la propiedad trasladada a dicho tiempo, es decir

$$
\operatorname{Pr}_{t_{i}}(p)=\operatorname{Tr}\left(\rho_{t_{0}} \Pi_{p, 0}\right), \quad \text { donde } \quad \Pi_{p, 0}=U\left(t_{0}, t_{i}\right) \Pi_{p} U\left(t_{i}, t_{0}\right) .
$$

Por lo tanto, en la representación de Heisenberg, una propiedad $p$ del contexto $\mathcal{C}_{i}$ al tiempo $t_{i}$ está dada por

$$
\Pi_{p, 0}=\sum_{k_{i} \in \sigma_{p}} \Pi_{i, 0}^{k_{i}}
$$

donde los proyectores $\Pi_{i, 0}^{k_{i}}=U\left(t_{0}, t_{i}\right) \Pi_{i}^{k_{i}} U\left(t_{i}, t_{0}\right)$ representan la traslación temporal de las propiedades atómicas $p_{i}^{k_{i}}$ desde el tiempo $t_{i}$ hasta el tiempo $t_{0}$. Los proyectores $\Pi_{i, 0}^{k_{i}}$ también verifican las ecuaciones (2).

\subsection{Contextos generalizados}

La representación de Heisenberg de un contexto de propiedades $\mathcal{C}_{i}$ al tiempo $t_{i}$ sugiere una posible generalización de la mecánica cuántica que permita asignar probabilidades a conjunciones de propiedades pertenecientes a contextos de diferentes tiempos.

En la mecánica cuántica ordinaria no es posible considerar propiedades de diferentes contextos, a menos que sus respectivas propiedades estén representadas por proyectores que conmuten entre sí. Lo mismo sucede si se quiere considerar contextos de propiedades a distintos tiempos. No es posible considerar simultáneamente propiedades de contextos de diferentes tiempos sin imponer condiciones adicionales.

El formalismo de Contextos Generalizados se propone considerar conjunciones de propiedades a distintos tiempos. Para esto impone un conjunto de restricciones a los contextos de propiedades a distintos tiempos que pueden considerarse simultáneamente. Dados los contextos de propiedades $\mathcal{C}_{1}, \ldots, \mathcal{C}_{n}$, correspondientes a los $n$ tiempos $t_{1}<\cdots<t_{n}$, los proyectores que representan a las propiedades de cada contexto deben satisfacer:

$$
\left[\Pi_{i, 0}^{k_{i}} ; \Pi_{j, 0}^{k_{j}}\right]=0, \quad i, j=1, \ldots, n, \quad k_{i} \in \sigma_{i}, \quad k_{j} \in \sigma_{j} .
$$


Es decir, los proyectores que representan las propiedades de cada contexto, al ser trasladados a un tiempo común $t_{0}$, deben conmutar entre sí. Esto es equivalente a decir que las propiedades estén representadas por proyectores que conmutan en la representación de Heisenberg. A estas condiciones se las denomina condiciones de compatibilidad.

Si se satisfacen las condiciones de compatibilidad, entonces los proyectores $\Pi_{0}^{\boldsymbol{k}}=\Pi_{1,0}^{k_{1}} \ldots \Pi_{n, 0}^{k_{n}}$, con $\boldsymbol{k}=\left(k_{1}, \ldots, k_{n}\right)$ y $k_{i} \in \sigma_{i}$, forman una descomposición proyectiva del espacio de Hilbert $\mathcal{H}$, ya que verifican

$$
\sum_{\boldsymbol{k}} \Pi_{0}^{\boldsymbol{k}}=I, \quad \Pi_{0}^{\boldsymbol{k}} \Pi_{0}^{\boldsymbol{k}^{\prime}}=\delta_{\boldsymbol{k} \boldsymbol{k}^{\prime}} \Pi_{0}^{\boldsymbol{k}}, \quad \boldsymbol{k}, \boldsymbol{k}^{\prime} \in \sigma_{1} \times \ldots \times \sigma_{n} .
$$

Luego, considerando sumas arbitrarias de los proyectores $\Pi_{0}^{\boldsymbol{k}}$, se obtiene un nuevo contexto que permite representar secuencias de propiedades a distintos tiempos. A este contexto se lo denomina contexto generalizado y sus proyectores están dados por

$$
\Pi_{p}=\sum_{k \in \sigma_{p}} \Pi_{0}^{k}
$$

donde $\boldsymbol{\sigma}_{\boldsymbol{p}} \subseteq \sigma_{1} \times \ldots \times \sigma_{n}$.

Los elementos de un contexto generalizado representan secuencias de propiedades a distintos tiempos, también denominadas historias cuánticas. Cada proyector atómico $\Pi_{0}^{k}=\Pi_{1,0}^{k_{1}} \ldots \Pi_{n, 0}^{k_{n}}$ representa a una historia cuántica atómica $\boldsymbol{p}^{\boldsymbol{k}}$ de la forma:

$$
\boldsymbol{p}^{k}=p_{1}^{k_{1}} \text { al tiempo } t_{1} y \ldots \text { y } p_{n}^{k_{n}} \text { al tiempo } t_{n} .
$$

En general, un proyector $\Pi_{\boldsymbol{p}}=\sum_{\boldsymbol{k} \in \boldsymbol{\sigma}_{\boldsymbol{p}}} \Pi_{0}^{\boldsymbol{k}}$ representa a una historia cuántica $\boldsymbol{p}$ formada por la disyunción de las historias atómicas $\boldsymbol{p}^{\boldsymbol{k}}$, con $\boldsymbol{k} \in \boldsymbol{\sigma}_{\boldsymbol{p}}$.

Como los contextos generalizados son reticulados ortocomplementados y distributivos, admiten una función de probabilidad. El formalismo de Contextos Generalizados define la probabilidad de una historia cuántica a partir de una generalización de la regla de Born. Dada una historia cuántica $\boldsymbol{p}$, representada por el proyector $\Pi_{\boldsymbol{p}}=\sum_{\boldsymbol{k} \in \boldsymbol{\sigma}_{\boldsymbol{p}}} \Pi_{0}^{\boldsymbol{k}}$, su probabilidad está dada por

$$
\operatorname{Pr}(\boldsymbol{p})=\operatorname{Tr}\left(\rho_{t_{0}} \Pi_{\boldsymbol{p}}\right)
$$

En las siguientes dos secciones se aplicará el formalismo de Contextos Generalizados para describir el proceso de medición y se analizarán los problemas mencionados en la sección 2 .

\section{El problema de la lectura definida en el formalismo de Contextos Generalizados}

En esta sección se aplica el formalismo de Contextos Generalizados para describir el proceso de medición en la mecánica cuántica y se analiza el problema de la lectura definida.

Consideremos nuevamente el escenario del proceso de medición descripto en la sección 2 . Sea $S+A$ el sistema compuesto, donde $S$ es el sistema a medir y $A$ el aparato de medición. En el tiempo $t_{1}$ comienza el proceso de medición y en el tiempo $t_{2}$ finaliza. El operador de evolución temporal $U\left(t_{2}, t_{1}\right)$ satisface

$$
\left|q_{i}\right\rangle\left|a_{0}\right\rangle \stackrel{U\left(t_{2}, t_{1}\right)}{\longrightarrow}\left|\phi_{i}\right\rangle\left|a_{i}\right\rangle \text {. }
$$

El estado inicial del sistema compuesto es $\left|\Psi_{t_{1}}\right\rangle=|\varphi\rangle\left|a_{0}\right\rangle$, donde $|\varphi\rangle=\sum_{i} c_{i}\left|q_{i}\right\rangle$. El estado final está dado por $\left|\Psi_{t_{2}}\right\rangle=\sum_{i} c_{i}\left|q_{i}\right\rangle\left|a_{i}\right\rangle$. 
El estado final es una combinación lineal de estados definidos del aparato de medición. Sin embargo, esto no es un problema para el formalismo de Contextos Generalizados, pues en este formalismo se abandona la idea de que un observable de un sistema físico tiene un valor definido únicamente si el vector de estado coincide con algún autovector del operador asociado a dicho observable.

Según este enfoque, el vector de estado que surge de resolver la ecuación de Schrödinger no describe las propiedades actuales del sistema, sino que determina las probabilidades de que las propiedades posibles se actualicen.

Esta interpretación del estado del sistema evita el problema de la lectura definida. No obstante, aún es necesario mostrar cómo este formalismo describe las correlaciones entre las propiedades observadas del aparato $A$ en el tiempo $t_{2}$ y las propiedades a medir del sistema $S$ en el tiempo $t_{1}$.

Para esto, primero se debe definir cuáles son los contextos de propiedades relevantes en cada tiempo. En el tiempo $t_{1}$ se consideran a las propiedades del sistema $S$ que se corresponden con los distintos valores posibles del observable $Q$, en conjunción con la propiedad asociada a que el aparato $A$ esté en el cero de la variable indicadora. En el espacio de Hilbert del sistema compuesto $\mathcal{H}_{S A}$, cada una de estas propiedades se representa con el proyector:

$$
\hat{\Pi}_{q_{j}}=\left|q_{j}\right\rangle\left\langle q_{j}|\otimes| a_{0}\right\rangle\left\langle a_{0}\right| .
$$

Para obtener una descomposición proyectiva del espacio de Hilbert $\mathcal{H}_{S A}$ hace falta agregar el proyector

$$
\hat{\Pi}_{q_{0}}=\hat{I}-\hat{I}_{S} \otimes\left|a_{0}\right\rangle\left\langle a_{0}\right| .
$$

De esta forma se verifican las siguientes relaciones:

$$
\sum_{j} \hat{\Pi}_{q_{j}}=\hat{I}, \quad \hat{\Pi}_{q_{j}} \hat{\Pi}_{q_{i}}=\delta_{i j} \hat{\Pi}_{q_{j}} .
$$

Los proyectores representan un conjunto de propiedades exhaustivas y mutuamente excluyentes del sistema total.

En el tiempo $t_{2}$ se consideran a las propiedades del aparato de medición $A$ correspondientes a los valores determinados de su variable indicadora, que se representan con los siguientes proyectores:

$$
\hat{\Pi}_{a_{j}}=\hat{I}_{S} \otimes\left|a_{j}\right\rangle\left\langle a_{j}\right| \text {. }
$$

Estos proyectores también forman una descomposición proyectiva del espacio de Hilbert $\mathcal{H}_{S A}$, pues

$$
\sum_{j} \hat{\Pi}_{a_{j}}=\hat{I}, \quad \hat{\Pi}_{a_{j}} \hat{\Pi}_{a_{i}}=\delta_{i j} \hat{\Pi}_{a_{j}} .
$$

Según el formalismo de Contextos Generalizados, la condición para que dos contextos de propiedades a distintos tiempos sean compatibles, y puedan formar parte de un mismo contexto generalizado, es que sus correspondientes proyectores trasladados a algún tiempo común $t_{0}$ conmuten entre sí.

En este caso es conveniente elegir $t_{0}=t_{1}$. Los proyectores de ambos contextos, al ser trasladados al tiempo $t_{1}$, se convierten en

$$
\hat{\Pi}_{q_{i}}^{(1)}=\hat{\Pi}_{q_{i}}, \quad \hat{\Pi}_{a_{j}}^{(1)}=\hat{U}\left(t_{1}, t_{2}\right) \hat{\Pi}_{a_{j}} \hat{U}^{-1}\left(t_{1}, t_{2}\right), \quad \hat{U}\left(t_{1}, t_{2}\right)=e^{-i \hat{H}\left(t_{1}-t_{2}\right) / \hbar}
$$

Vanni y Laura (2013) han demostrado que estos proyectores trasladados temporalmente conmutan entre sí, es decir 


$$
\left[\hat{\Pi}_{q_{i}}^{(1)}, \hat{\Pi}_{a_{j}}^{(1)}\right]=0
$$

Por lo tanto, es posible construir un contexto generalizado que involucra en el tiempo $t_{1}$ a las propiedades del sistema $S$, representadas por los proyectores

$$
\hat{\Pi}_{q_{j}}=\left|q_{j}\right\rangle\left\langle q_{j}|\otimes| a_{0}\right\rangle\left\langle a_{0}\right| \text { у } \hat{\Pi}_{q_{0}}=\hat{I}-\hat{I} \otimes\left|a_{0}\right\rangle\left\langle a_{0}\right|,
$$

y en el tiempo $t_{2}$ a las propiedades del aparato de medición, representadas por los proyectores

$$
\hat{\Pi}_{a_{j}}=\hat{I}_{S} \otimes\left|a_{j}\right\rangle\left\langle a_{j}\right| .
$$

En este contexto generalizado es posible calcular la probabilidad de que el sistema a medir haya tenido al tiempo $t_{1}$ la propiedad $q_{j}$, condicional a que al tiempo $t_{2}$ el resultado que indica el aparato sea $a_{j}$. La probabilidad se obtiene de la siguiente manera (Vanni \& Laura 2013):

$$
\operatorname{Pr}\left\{\left(q_{j}, t_{1}\right) \mid\left(a_{j}, t_{2}\right)\right\}=\frac{\operatorname{Pr}\left\{\left(q_{j}, t_{1}\right) \wedge\left(a_{j}, t_{2}\right)\right\}}{\operatorname{Pr}\left\{\left(a_{j}, t_{2}\right)\right\}}=\frac{\left\langle\Psi_{t_{1}}\left|\hat{\Pi}_{q_{j}}^{(1)} \hat{\Pi}_{a_{j}}^{(1)}\right| \Psi_{t_{1}}\right\rangle}{\left\langle\Psi_{t_{1}}\left|\hat{\Pi}_{a_{j}}^{(1)}\right| \Psi_{t_{1}}\right\rangle}=1
$$

Podría sospecharse de que el resultado depende de haber elegido $t_{0}=t_{1}$ como tiempo común, pues en dicho tiempo el estado del sistema es $\left|\Psi_{t_{1}}\right\rangle=|\varphi\rangle\left|a_{0}\right\rangle$, un estado en que $S$ y $A$ aún no están correlacionados. Sin embargo, el tiempo de referencia $t_{0}$ es arbitrario en el formalismo de contextos generalizados, se podría usar $t_{0}=t_{2}$ y se obtendría el mismo resultado, esta vez involucrando en el cálculo al estado $\left|\Psi_{t_{2}}\right\rangle$,

$$
\operatorname{Pr}\left\{\left(q_{j}, t_{1}\right) \mid\left(a_{j}, t_{2}\right)\right\}=\frac{\operatorname{Pr}\left\{\left(q_{j}, t_{1}\right) \wedge\left(a_{j}, t_{2}\right)\right\}}{\operatorname{Pr}\left\{\left(a_{j}, t_{2}\right)\right\}}=\frac{\left\langle\Psi_{t_{2}}\left|\hat{\Pi}_{q_{j}}^{(2)} \hat{\Pi}_{a_{j}}^{(2)}\right| \Psi_{t_{2}}\right\rangle}{\left\langle\Psi_{t_{2}}\left|\hat{\Pi}_{a_{j}}^{(2)}\right| \Psi_{t_{2}}\right\rangle}=1,
$$

donde $\hat{\Pi}_{q_{j}}^{(2)}=\hat{U}\left(t_{2}, t_{1}\right) \hat{\Pi}_{q_{j}} \hat{U}\left(t_{1}, t_{2}\right)$ y $\hat{\Pi}_{a_{j}}^{(2)}=\hat{\Pi}_{a_{j}}$.

El resultado igual a uno de esta probabilidad condicional se interpreta físicamente de la siguiente manera: si la aguja del aparato $A$ indica $a_{j}$ en el tiempo $t_{2}$, entonces en el tiempo $t_{1}$ el sistema $S$ tenía la propiedad $q_{j}$ y el aparato $A$ se encontraba en el cero de la variable indicadora.

Esta interpretación de la probabilidad condicional está de acuerdo con lo que se observa en el laboratorio y el estado final $\left|\Psi_{t_{2}}\right\rangle=\sum_{i} c_{i}\left|q_{i}\right\rangle\left|a_{i}\right\rangle$, a pesar de ser una superposición, no genera ningún efecto no deseable.

De esta forma, el formalismo de Contextos Generalizados demuestra que luego de la medición se obtiene una correlación entre las propiedades del aparato al tiempo $t_{2}$ y las del sistema medido al tiempo $t_{1}$.

En la siguiente sección se aplica este formalismo para analizar el problema de la base privilegiada.

\section{El problema de la base privilegiada en el formalismo de Contextos Generalizados}


La respuesta al problema de la lectura definida ha dependido de la elección de un contexto generalizado particular: aquel que se construye a partir de considerar el contexto de propiedades $q_{i}$ al tiempo $t_{1}$ y el contexto de propiedades $a_{j}$ al tiempo $t_{2}$.

Sin embargo, en cada tiempo es posible considerar diferentes contextos de propiedades. En particular, en el tiempo $t_{2}$ es posible elegir otro contexto, cuyas propiedades no se correspondan con que la variable puntero tenga valores definidos. Esta libertad es responsable de la ambigüedad en la elección de la base de la medición y da lugar al problema de la base privilegiada.

En el caso de la mecánica cuántica ordinaria, el problema se circunscribe a un único tiempo. En cambio, en el formalismo de Contextos Generalizados en principio se podrían elegir diferentes bases o contextos de propiedades en ambos tiempos.

Se han propuesto diferentes soluciones al problema de la elección de la base del aparato de medición. Algunos sostienen que debe agregarse a la mecánica cuántica un postulado adicional que privilegie a una base con respecto a las otras. Esta posición es compartida por las interpretaciones modales de la mecánica cuántica (Lombardi \& Castagnino 2008). Otra solución es la propuesta por Zurek (1981), que sostiene que el proceso de decoherencia basta para seleccionar a la base de la variable puntero del aparato de medición. Por último, están quienes sostienen que la libertad de elección de los contextos de propiedades es una característica esencial de la mecánica cuántica que debe ser aceptada (Griffiths 2013). La elección de la base se debe hacer de acuerdo con motivos de utilidad según el proceso físico que se quiere describir. En la actualidad conviven las tres posturas y la discusión que continúa abierta.

El enfoque de Contextos Generalizados adopta la tercera postura. Para cada proceso físico la elección del contexto generalizado se realiza teniendo en cuenta su utilidad para la descripción de dicho proceso. En el caso de la medición, al finalizar el proceso se obtiene un valor definido de la variable puntero del aparato, por lo tanto, se debe elegir un contexto generalizado que contenga a las propiedades que se correspondan con los valores definidos de la variable puntero.

De esta forma se resuelve el problema de la elección de la base en el tiempo $t_{2}$ en el formalismo de Contextos Generalizados. No obstante, aún puede plantearse la pregunta sobre la elección del contexto de propiedades al tiempo $t_{1}$. ¿Es posible en el tiempo $t_{1}$ elegir otro contexto de propiedades distinto al correspondiente al observable $Q$ ? Es decir, resta indagar si este formalismo permite o no que un mismo aparato de medición pueda medir distintos observables cambiando la elección del contexto generalizado. Se demostrará a continuación que esto no es posible.

Sea un vector cualquiera $|x\rangle=\sum_{i}\left|q_{i}\right\rangle\left\langle q_{i} \mid x\right\rangle \in \mathcal{H}_{S}$ y sea $x$ la propiedad correspondiente a que el sistema tiene la propiedad representada por el proyector $|x\rangle\langle x|$ y el aparato se encuentra listo para la medición. El proyector que representa a la propiedad $x$ está dado por

$$
\hat{\Pi}_{x}=|x\rangle\left\langle x|\otimes| a_{0}\right\rangle\left\langle a_{0}\right| .
$$

Interesa saber si el aparato $A$ puede medir a la propiedad $x$. Para que esto sea posible la propiedad $x$ al tiempo $t_{1}$ y las propiedades representadas por los proyectores $\hat{\Pi}_{a_{j}}=\hat{I}_{S} \otimes\left|a_{j}\right\rangle\left\langle a_{j}\right|$ al tiempo $t_{2}$ deben poder ser incluidas en un mismo contexto generalizado. Para esto es necesario que los proyectores de ambos contextos conmuten al ser trasladados a un tiempo común $t_{0}$. Si se elige $t_{0}=t_{2}$, entonces se debe satisfacer

$$
\left[\hat{U}\left(t_{2}, t_{1}\right) \hat{\Pi}_{x} \hat{U}\left(t_{1}, t_{2}\right), \hat{\Pi}_{a_{k}}\right]=0
$$

para todo $\hat{\Pi}_{a_{k}}$. 
Se debe notar que

$$
\hat{U}\left(t_{2}, t_{1}\right) \hat{\Pi}_{x} \hat{U}\left(t_{1}, t_{2}\right)=\sum_{i, j}\left\langle q_{i} \mid x\right\rangle\left\langle x \mid q_{j}\right\rangle\left|\phi_{i}\right\rangle\left\langle\phi_{j}|\otimes| a_{i}\right\rangle\left\langle a_{j}\right| .
$$

Por lo tanto:

$$
\begin{gathered}
\hat{U}\left(t_{2}, t_{1}\right) \hat{\Pi} \hat{U}_{x}\left(t_{1}, t_{2}\right) \hat{\Pi}_{a_{k}}=\sum_{i}\left\langle q_{i} \mid x\right\rangle\left\langle x \mid q_{k}\right\rangle\left|\phi_{i}\right\rangle\left\langle\phi_{k}|\otimes| a_{i}\right\rangle\left\langle a_{k}\right|, \\
\hat{\Pi}_{a_{k}} \hat{U}\left(t_{2}, t_{1}\right) \hat{\Pi_{x}} \hat{U}\left(t_{1}, t_{2}\right)=\sum_{j}\left\langle q_{k} \mid x\right\rangle\left\langle x \mid q_{j}\right\rangle\left|\phi_{k}\right\rangle\left\langle\phi_{j}|\otimes| a_{k}\right\rangle\left\langle a_{j}\right| .
\end{gathered}
$$

Luego, para que se satisfaga la ecuación (3) para todo $\hat{\Pi}_{a_{k}}$, debe pasar que $|x\rangle=\left|q_{l}\right\rangle$ para algún $\left|q_{l}\right\rangle$ de los que forman la base del observable $Q$. Por lo tanto, no es posible que el aparato $A$ mida una propiedad que no se corresponda con el observable $Q$.

\section{Conclusiones}

En este trabajo se ha analizado el proceso de medición en la mecánica cuántica. La formulación ortodoxa de la mecánica cuántica distingue dos tipos de evoluciones temporales: la evolución por colapso y la evolución de Schrödinger. La primera se aplica a los procesos de medición y la segunda a los procesos físicos ordinarios. La distinción tajante entre dos tipos de procesos físicos, con sus respectivas evoluciones temporales, no es satisfactoria desde un punto de vista teórico. Es deseable una teoría cuántica que describa a ambos tipos de procesos de la misma manera, utilizando únicamente la evolución de Schrödinger.

En la sección 2 se utilizó el formalismo de la mecánica cuántica ortodoxa para describir el proceso de medición como un proceso de interacción ordinario entre un sistema a medir y un aparato de medición, con su evolución temporal dada únicamente por la ecuación de Schrödinger. Se presentaron los dos problemas centrales que aparecen en esta descripción: el problema de la lectura definida y el problema de la base privilegiada.

El primero consiste en explicar cómo se obtiene un valor definido de la variable puntero, si el estado del sistema se encuentra en una superposición de autovectores del observable del aparato. El segundo aparece debido a que la descomposición del estado final no es única, sino que depende de la base elegida. Por lo tanto, se debe explicar cómo se elige la base en la cual el sistema adquiere un valor definido.

En las siguientes secciones se analizaron los problemas de la medición utilizando una teoría de historias cuánticas denominada formalismo de Contextos Generalizados. Primero, en la sección 3, se resumieron las ideas centrales del formalismo. Entre sus características principales se debe destacar que los procesos de medición son tratados de la misma forma que el resto de los procesos físicos. La medición pierde su estatus privilegiado y pasa a ser descripta como una interacción física ordinaria entre un sistema a medir y un aparato de medición. Su evolución temporal es descripta únicamente a través de la evolución de Schrödinger, no se hace uso del postulado de colapso. Además, se extiende el formalismo estándar de la mecánica cuántica de modo tal de poder definir operaciones lógicas entre propiedades a distintos tiempos. Por lo tanto, la medición se puede describir como una correlación de propiedades del sistema compuesto a distintos tiempos.

Luego, en la sección 4 y en la sección 5, se aplicó el formalismo de Contextos Generalizados para analizar los problemas de la lectura definida y la base privilegiada, respectivamente. 
En la sección 4 se mostró que no es un problema que el estado del sistema compuesto, después de la medición, sea una combinación lineal de estados del aparato. Esto se debe a que en este formalismo el vector de estado no describe las propiedades actuales del sistema, sino que determina las probabilidades de que las propiedades posibles se actualicen.

Además, se demostró que es posible construir un contexto generalizado que incluya en el tiempo $t_{1}$ a las propiedades $q_{j}$ correspondientes al observable medido del sistema $S$ y en el tiempo $t_{2}$ a las propiedades $a_{j}$ correspondientes a la variable puntero del aparato. Usando este contexto generalizado se mostró que la probabilidad de que el sistema $S$ haya tenido la propiedad $q_{j}$ en el tiempo $t_{1}$, dado que el aparato $A$ indicó $a_{j}$ en el tiempo $t_{2}$, es igual a uno. De esta forma, el formalismo de Contextos Generalizados demuestra que, luego de la medición, se obtiene una correlación entre las propiedades del aparato al tiempo $t_{2}$ y las del sistema $S$ al tiempo $t_{1}$.

En la sección 5 se discutió el problema de la base privilegiada. Según el formalismo de Contextos Generalizados, para cada proceso físico la elección del contexto generalizado se realiza teniendo en cuenta su utilidad para la descripción de dicho proceso. En el caso de la medición, al finalizar el proceso se obtiene un valor definido de la variable puntero del aparato, por lo tanto, se debe elegir un contexto generalizado que contenga a las propiedades que se correspondan con los valores definidos de la variable puntero.

De esta forma se resuelve el problema de la elección de la base en el tiempo $t_{2}$. No obstante, aún puede plantearse la pregunta sobre la elección del contexto de propiedades al tiempo $t_{1}$. Es decir, si es posible que un mismo aparato de medición pueda medir distintos observables cambiando la elección del contexto generalizado. Al final de la sección 5 se demostró que esto no es posible: el aparato $A$ puede medir un único observable.

Bibliografía

Ballentine, L. (1998), Quantum Mechanics. A Modern Development, Singapur: World Scientific.

Cohen, D. (1989), An Introduction to Hilbert Space and Quantum Logic, Nueva York: Springer-Verlag.

Gell-Mann, M. y J. B. Hartle (1990), “Quantum Mechanics in the Light of Quantum Cosmology”, en Zurek, W. (ed.), Complexity, Entropy and the Physics of Information, Reading: Addison-Wesley, vol. VIII.

Griffiths, R. (1984), “Consistent Histories and the Interpretation of Quantum Mechanics”, Journal of Statistical Physics 36: 219-272.

Griffiths, R. (2002), Consistent Quantum Theory, Cambridge: Cambridge University Press.

Griffiths, R. (2013), “A Consistent Quantum Ontology”, Studies in History and Philosophy of Modern Physics 44: 93-114.

Laura, R. y L. Vanni (2008), “Conditional Probabilities and Collapse in Quantum Measurements”, International Journal of Theoretical Physics 47: 2382-2392.

Laura, R. y L. Vanni (2008), “Contexto de historias en la teoría de cuántica”, Epistemología e Historia de la Ciencia 14: 519-528.

Laura, R. y L. Vanni (2009), “Time Translation of Quantum Properties”, Foundations of Physics 39: 160-173.

Lombardi, O. y M. Castagnino (2008), “A Modal-Hamiltonian Interpretation of Quantum Mechanics”, Studies in History and Philosophy of Modern Physics 39: 380-443.

Losada, M., Vanni, L. y R. Laura (2013), "Probabilities for Time Dependent Properties in Classical and Quantum Mechanics”, Physical Review A 87: \# 052128. 
96 | Marcelo Losada y Roberto Laura

Mittelstaedt, P. (1998), The Interpretation of Quantum Mechanics and the Measurement Process, Cambridge: Cambridge University Press.

Omnès, R. (1988), “Logical Reformulation of Quantum Mechanics. I. Foundations”, Journal of Statistical Physics 53: 893-932.

Omnés, R. (1994), The Interpretation of Quantum Mechanics, Princeton: Princeton University Press.

Omnès, R. (1999), Understanding Quantum Mechanics, Princeton: Princeton University Press.

Vanni, L. y R. Laura (2013), “The Logic of Quantum Measurements”, International Journal of Theoretical Physics 52: 2386-2394.

Zurek, W. H. (1981), “Pointer Basis of Quantum Apparatus: Into What Mixture does the Wave Packet Collapse?”, Physical Review D 24: 1516-1525. 